\title{
Cost-Effectiveness Analysis of Durvalumab Plus Chemotherapy in the First-Line Treatment of Extensive-Stage Small Cell Lung Cancer
}

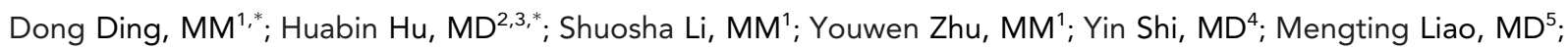
Jin Liu, $\mathrm{MD}^{6}$; Xu Tian, $\mathrm{MM}^{6}$; Aiting Liu, $\mathrm{MD}^{7}$; and Jin Huang, $\mathrm{PhD}^{1,8}$

\begin{abstract}
Background: In the CASPIAN trial, durvalumab + chemotherapy demonstrated significant improvements in overall survival compared with chemotherapy alone in patients with extensive-stage small cell lung cancer (SCLC). We aimed to assess the cost-effectiveness of durvalumab in patients with extensive-stage SCLC from the US healthcare system perspective. Patients and Methods: A comprehensive Markov model was adapted to evaluate cost and effectiveness of durvalumab combination versus platinum/etoposide alone in the first-line therapy of extensive-stage SCLC based on data from the CASPIAN study. The main endpoints included total costs, life years $(\mathrm{LYs})$, quality-adjusted life-years (QALYs), and incremental coste-ectiveness ratios (ICERs). Model robustness was assessed with sensitivity analysis, and additional subgroup analyses were also performed. Results: Durvalumab + chemotherapy therapy resulted in an additional 0.27 LYs and 0.20 QALYs, resulting in an ICER of $\$ 464,711.90$ per QALY versus the chemotherapy treatment. The cost of durvalumab has the greatest influence on this model. Subgroup analyses showed that the ICER remained higher than $\$ 150,000$ / QALY (the willingness-to-pay threshold in the United States) across all patient subgroups. Conclusions: Durvalumab in combination with platinum/etoposide is not a cost-effective option in the first-line treatment of patients with extensive-stage SCLC.
\end{abstract}

J Nat I Compr Canc Netw 2021;19(10):1141-1147 doi: $10.6004 /$ jnccn.2020.7796

\footnotetext{
${ }^{1}$ Department of Oncology, Xiangya Hospital, Central South University, Changsha, Hunan; ${ }^{2}$ Department of Medical Oncology, The Sixth Affiliated Hospital of Sun Yat-sen University, Guangzhou; ${ }^{3}$ Guangdong Institute of Gastroenterology, Guangdong Provincial Key Laboratory of Colorectal and Pelvic Floor Diseases, Guangzhou; ${ }^{4}$ Department of Pharmacy, and ${ }^{5}$ Department of Health Management Center, Xiangya Hospital, Central South University, Changsha, Hunan; ${ }^{6}$ School of Computer Science and Engineering, Central South University, Changsha; ${ }^{7}$ Hunan Healthcare Security Administration, Changsha; and ${ }^{8}$ Department of Dermatology, Hunan Engineering Research Center of Skin Health and Disease, Hunan Key Laboratory of Skin Cancer and Psoriasis, Xiangya Hospital, Central South University, Changsha, Hunan, China.

*These authors contributed equally to this study.
}

\section{Background}

Lung cancer remains the leading cause of cancer incidence and mortality, with 2.1 million new lung cancer cases and 1.8 million deaths in the United States. ${ }^{1,2}$ Approximately two-thirds of patients with small cell lung cancer (SCLC) have extensive-stage disease at diagnosis, with a 5-year survival rate of $<7 \%{ }^{3,4}$ Etoposide with either cisplatin or carboplatin (platinum/etoposide) was the standard first-line therapy for extensive-stage SCLC for 3 decades. ${ }^{5,6}$ Most patients experience relapse within 6 months of completing initial treatment, despite high rates of response to chemotherapy. ${ }^{7,8}$

Immunotherapy targeting PD-L1 has demonstrated clinical activity in first-line treatment of extensive-stage SCLC. ${ }^{9}$ Durvalumab is a selective, high-affinity human IgG1 monoclonal antibody that blocks PD-L1 binding to PD- 1 and CD $80 .{ }^{10}$ Recently, a randomized, controlled, open-label, phase III trial (CASPIAN) compared durvalumab + chemotherapy versus chemotherapy alone in the first-line treatment of patients with extensive-stage SCLC. The results demonstrated that treatment with durvalumab + platinum/ etoposide significantly improved overall survival (OS; hazard ratio $[\mathrm{HR}], 0.73 ; 95 \% \mathrm{CI}, 0.59-0.91 ; P<.0001) .{ }^{11}$ Durvalumab + platinum/etoposide as the first-line treatment option for patients with extensive-stage SCLC was recommended in the NCCN Clinical Practice Guidelines in Oncology (NCCN Guidelines) for SCLC, Version $2.2020,{ }^{12}$ and was subsequently approved by the FDA in March 2020. ${ }^{13}$

Although the combination of durvalumab + chemotherapy provides clinical benefits, these benefits must be weighed against the financial burden. The current study investigated the economic outcomes of durvalumab + chemotherapy versus chemotherapy alone for patients with previously untreated extensive-stage SCLC in the

See JNCCN.org for supplemental online content. 
context of the US healthcare system by using the published results of the phase III CASPIAN trial.

\section{Patients and Methods}

\section{Model Structure}

A comprehensive Markov model with 3 mutually exclusive health states-progression-free survival (PFS), progressive disease (PD), and death-was adapted to evaluate the costs and effectiveness of treatment of extensive-stage SCLC with durvalumab + chemotherapy and chemotherapy alone. All patients were assumed to be treated with durvalumab + chemotherapy or chemotherapy alone in the PFS state until disease progression. When the disease progressed or unacceptable adverse reactions occurred, patients in both groups could receive subsequent comprehensive treatment until death (supplemental eFigure 1, available with this article at JNCCN. org).

We assigned the model cycle as 6 weeks, to fit with the various dosage frequencies of interventions. The time horizon was lifetime. An annual 3\% discount rate was adopted both on costs and outcomes. ${ }^{14}$ The primary outcome measures of the models included the total costs, life-years (LYs), quality-adjusted life-years (QALYs), and incremental cost-effectiveness ratios (ICERs). Based primarily on results of the CASPIAN trial, the model structure and data were also supplemented by data retrieved from publicly available databases and published literature. The model was constructed via TreeAge Pro 2020 (TreeAge Software, Inc.).

\section{Model Transitions and Survival Estimates}

The transition probabilities for both groups were derived from the PFS and OS curves in the CASPIAN trial. We used GetData Graph Digitizer 2.26 to collect data points from the curves for the 2 groups based on the method described by Rini et al, ${ }^{15}$ and these data points were then fit into the parametric survival model. The Weibull distribution provided the most appropriate fit to pseudoindividual patient data among the exponential, loglogistic, log-normal, and Gompertz distributions, according to the Akaike information criterion and the Bayesian information criterion, combined with visual inspection. ${ }^{16}$ A detailed description of the survival model selection is shown in supplemental eTable 1 and eFigure 2. Detailed parameters of the survival model are shown in Table 1.

\section{Utility Estimates}

We used utility to measure patients' health-related quality of life at a particular health state. The mean health utility values for the PFS and PD states, $0.840^{17,18}$ and $0.473,{ }^{19}$ respectively, were derived from the previously published literature. The disutility values due to grade $3 / 4$ adverse effects (AEs) were factored during the analysis. ${ }^{19,20}$

\section{Cost Inputs}

This analysis adopted the third-party payer in the United States, which only considered direct medical care costs, including costs of drugs, administration, ${ }^{21}$ AEs (assuming that AEs appeared only once in the PFS and PD states), and laboratory ${ }^{22}$ and radiographic tests. ${ }^{23}$ According to the CASPIAN trial, the durvalumab + chemotherapy group received up to 4 cycles of durvalumab at 1,500 $\mathrm{mg}$ and chemotherapy (etoposide at $80-100 \mathrm{mg} / \mathrm{m}^{2}$ [administered on days $1-3$ of each 21-day cycle] and either carboplatin at an area under the curve [AUC] of $5-6 \mathrm{mg} / \mathrm{mL}$ or cisplatin at $75-80 \mathrm{mg} / \mathrm{m}^{2}$ [administered on day 1 of each cycle]). After 4 cycles, patients received maintenance treatment with $1,500 \mathrm{mg}$ of durvalumab administered intravenously every 4 weeks. Patients in the platinum/etoposide group could receive an additional 2 cycles of platinum/etoposide (up to 6 cycles total). For chemotherapy dosing, we used a standard AUC of $6 \mathrm{mg} / \mathrm{mL} / \mathrm{min}$ and assumed male sex, 65 years of age, weight of $70 \mathrm{~kg}$, height of $70 \mathrm{~cm}$, body surface area of $1.84 \mathrm{~m}^{2}$, and serum creatinine level of $1 \mathrm{mg} / \mathrm{dL}^{20}{ }^{20}$ Drug prices were based on the Centers for Medicare \& Medicaid Services Average Sales Price $^{24}$ (Table 1). The subsequent therapy received in 2 groups were not well described in the CASPIAN trial. Therefore, we assumed patients received the standard second-line chemotherapy: topotecan alone $\left(1.5 \mathrm{mg} / \mathrm{m}^{2} / \mathrm{d}\right.$ administered intravenously on days $1-5$ every 21 days). Its dose was based on clinical trial data. ${ }^{25,26}$

Grade 3/4 severe AEs were included in the model, which had notably different probabilities between the arms of the CASPIAN trial. The incidence rates of AEs in the model came from the CASPIAN trial data. The costs of managing AEs per event in the United States were extracted from the published literature. ${ }^{27,28}$ All costs were adjusted to present 2020 US dollars and the US consumer price index was adopted as reference for inflation. Information on these costs is provided in Table 1.

\section{Sensitivity Analysis}

A series of sensitivity analyses were performed to evaluate the robustness of the model and the influence of variable uncertainty on the results. The 1-way sensitivity analyses were used to examine the individual effects of this parameter on the ICERs. The values, variation ranges, and distributions of the parameters are shown in Table 1. We also completed probabilistic sensitivity analyses to assess the probability of durvalumab + chemotherapy being costeffective by performing 10,000 Monte Carlo repetitions.

In addition, subgroup analyses were completed in patients in the forest plot of the CASPIAN trial based on the subgroup-specific HRs according to the approach taken by Hoyle et al. ${ }^{29}$ 


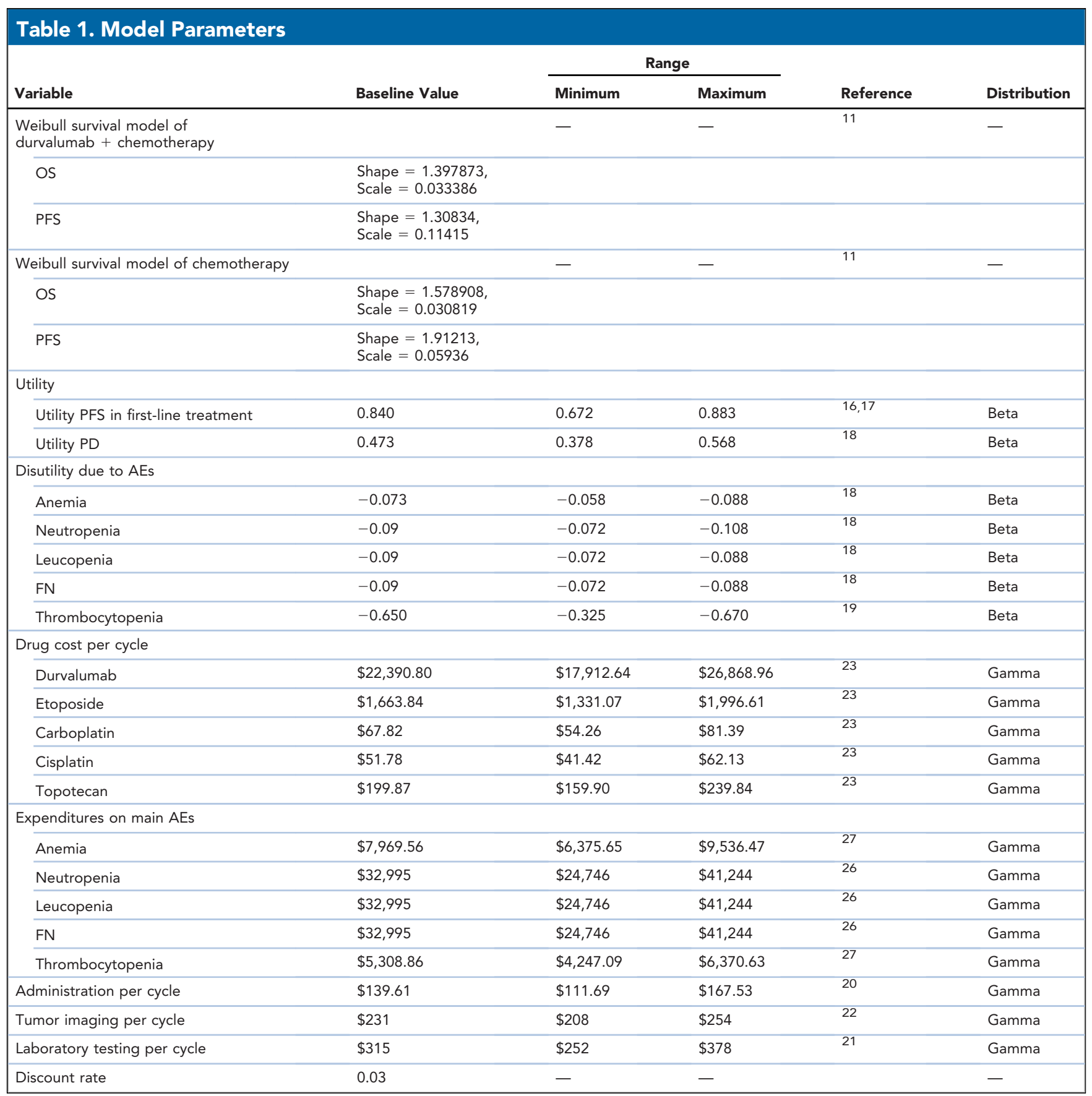

Abbreviations: AEs, adverse events; FN, febrile neutropenia; OS, overall survival; PD, progressive disease; PFS, progression-free survival.

\section{Results}

\section{Base Case Results}

For the patients with previously untreated extensive-stage SCLC, durvalumab + chemotherapy provided an additional 0.20 QALYs (0.27 LYs) at an incremental cost of $\$ 91,469.96$ compared with chemotherapy alone, resulting in an ICER value of $\$ 464,711.90$ per QALY $(\$ 345,458.24$ per LY) (Table 2).
Sensitivity Analysis

Results of the one-way sensitivity analyses are presented in the tornado diagrams (Figure 1). The greatest influence of variables on the ICER was the cost of durvalumab. Other considerable influential parameters were risk of neutropenia in chemotherapy group, risk of neutropenia in durvalumab group, and utility of PD.

Results of ICER scatterplot (supplemental eFigure 3) and acceptability curves (Figure 2) 


\section{Table 2. Baseline Results}

\begin{tabular}{|lll|}
\hline Parameters & $\begin{array}{l}\text { Durvalumab }+ \\
\text { Chemotherapy }\end{array}$ & Chemotherapy \\
\hline LYs & 2.20 & 1.93 \\
\hline QALYs & 1.45 & 1.25 \\
\hline Total cost, \$ & $\$ 164,508.07$ & $\$ 73,038.11$ \\
\hline ICER \$/LYa & $\$ 345,458.24$ & - \\
\hline ICER \$/QALY & $\$ 464,711.90$ & - \\
\hline WTP \$/QALY & $\$ 150,000$ & - \\
\hline
\end{tabular}

Abbreviations: ICER, incremental cost-effectiveness ratio; LY, life-year; QALY, quality-adjusted life-year; WTP, willingness-to-pay.

${ }^{a}$ Compared with chemotherapy (\$/LY).

${ }^{b}$ Compared with chemotherapy (\$/QALY).

demonstrated that the probability of durvalumab + chemotherapy being cost-effective, compared with that of chemotherapy alone was $0 \%$ at a WTP threshold of $\$ 150,000$. The ICER remained greater than the WTP threshold across all patient subgroups in the subgroup analyses (supplemental eTable 2)

\section{Discussion}

In recent years, immune checkpoint inhibitors (ICIs) have shown significant clinical benefits in patients with non-small cell lung cancer (NSCLC) at different stages, and there are also many studies on immunotherapy for refractory or metastatic SCLC. ${ }^{9,11,30-34}$ Although the results of a study showed that first-line immunotherapy (ipilimumab) + chemotherapy did not significantly improve the OS of patients with extensive-stage SCLC compared with chemotherapy alone ${ }^{34,35}$ some inspiring results of 2 phase III studies indicated that the addition of atezolizumab or durvalumab to chemotherapy showed promising clinical activity. ${ }^{9,11}$ Considering the expensive cost of ICIs, we conducted the first study to estimate the cost-effectiveness of durvalumab + chemotherapy versus chemotherapy alone as first-line therapy for patients with extensive-stage SCLC.

In our analysis, the base case results indicated that durvalumab + chemotherapy produced an additional 0.20 QALYs compared with chemotherapy alone, and the ICER was $\$ 464,711.90$, which was higher than $\$ 150,000$ / QALY (the WTP threshold in the United States). The acceptability curves also demonstrated that a paucity of certainty was achieved by durvalumab + chemotherapy at the threshold of $\$ 150,000$ in the United States. However, this treatment should not be disregarded, because the model outcomes are sensitive to the price of durvalumab in the 1-way sensitivity analyses. The ICER was still greater than $\$ 360,000 /$ QALY regardless of the variation within a certain range of the most sensitive variables, which was not considered to be cost-effective. Subgroup characteristics associated with cost-effectiveness suggest that brain or central nervous system metastases, female sex, current smoker status, and carboplatin use are

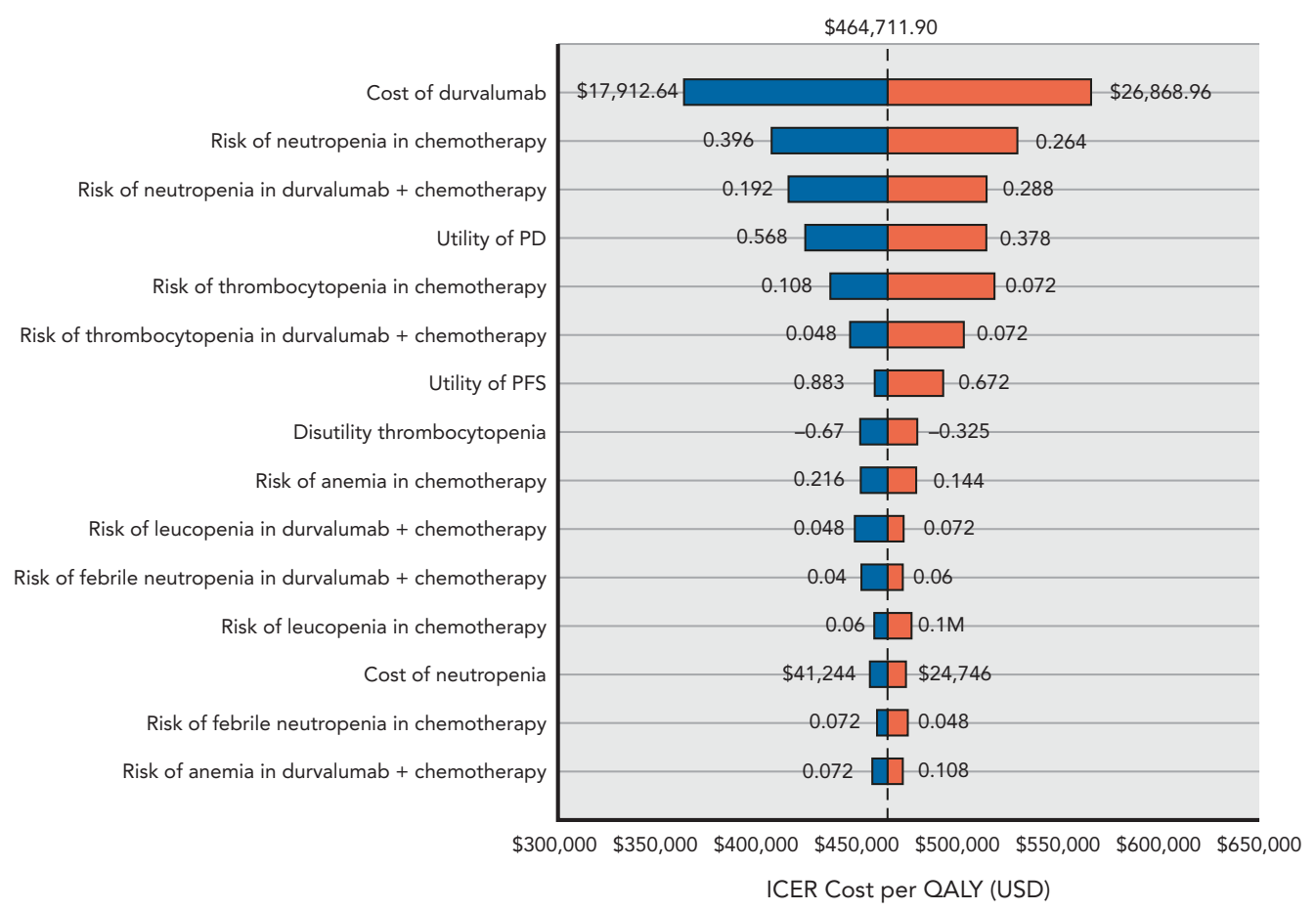

Figure 1. Tornado diagram for one-way sensitivity analysis.

Abbreviations: ICER, incremental cost-effectiveness ratio; QALY, quality-adjusted life years; PD, progressive disease; PFS, progression-free survival. 


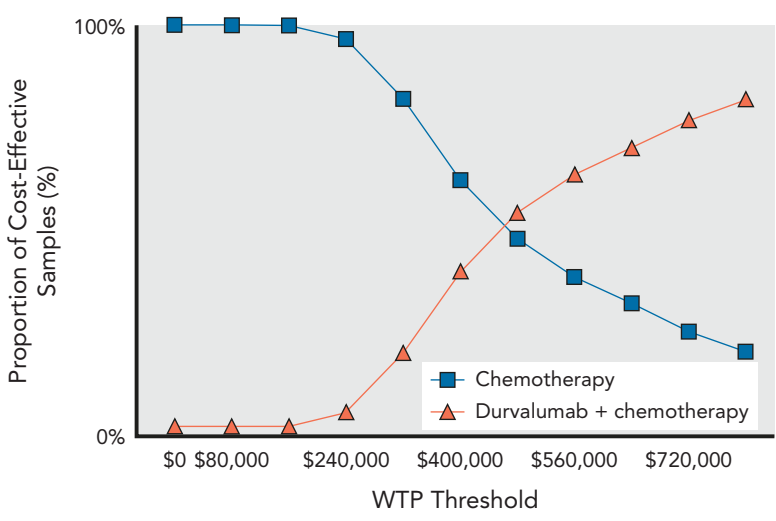

Figure 2. Acceptability curves for the choice of durvalumab + chemotherapy treatment strategies at different WTP thresholds in patients with small cell lung cancer.

Abbreviation: WTP, willingness-to-pay.

factors that should be considered to make durvalumab + chemotherapy more cost-effective. However, durvalumab + chemotherapy was not a cost-effective strategy across all patient subgroups.

The IMpower133 trial is another study that demonstrated that first-line treatment with immunotherapy (atezolizumab) + chemotherapy resulted in significantly longer OS and PFS compared with chemotherapy alone for patients with extensive-stage SCLC. Subsequently, the combination of atezolizumab and carboplatin/etoposide was approved by the FDA in this indication and was recommended in NCCN Guidelines for SCLC, Version 1.2019. ${ }^{36}$ A cost-effectiveness analysis reported by Zhou et $\mathrm{al}^{37}$ suggested that the addition of atezolizumab to carboplatin/etoposide is not a cost-effective choice in the first-line treatment of extensive-stage SCLC from an American perspective, with a gain of 0.10 QALYs and an ICER of $\$ 528,810$ per QALY. Our results were in agreement with this finding, and the increased QALYs of durvalumab + chemotherapy (0.20 QALYs) in our study were more significant than those of atezolizumab + chemotherapy. Similarly, immunotherapy combined with chemotherapy was not a cost-effective choice in the costeffectiveness analysis for NSCLC. ${ }^{38,39}$ The price of ICIs was always the greatest factor affecting the outcomes. A reduction in price of ICIs through trade-off negotiations regarding drug price and coverage may be an appropriate and effective method to improve the cost-effectiveness.

It is a challenge to approve new drugs by solely relying on survival benefits. That is why different economic elements in different regions should be taken into account during drug approval. Meanwhile, physicians and administrators should be aware of the significance of utilizing predictive markers in appropriate patient selection to map out cost-effective strategies aiming to sustain our healthcare systems.
PD-Ll expression has been regarded as a predictive biomarker of response to ICIs. In many pharmacoeconomic studies of NSCLC, it has been found that high PD-L1 expression incrementally improves QALYs, resulting in a decrease in ICER. ${ }^{28,40-42}$ Our previous analysis results attested that first-line treatment with pembrolizumab is a cost-effective strategy compared with platinumbased chemotherapy in patients with locally advanced or metastatic NSCLC and PD-L1 tumor proportion score (TPS) $\geq 50 \%$, but not in those with TPS $\geq 20 \%$ and $\geq 1 \%$. ${ }^{41}$ Another predictive biomarker of immunotherapy for lung cancer is high tumor mutational burden (TMB) which had no significant correlation with tumor PD-L1 expression. ${ }^{43-45}$ Similarly, it was found that there was an association between immunotherapy response and PD-L1 expression or TMB in SCLC. ${ }^{30,43,46}$ Unfortunately, the CASPIAN trial did not report the expression of PD-L1 or TMB. The ICER of durvalumab + chemotherapy may be improved by selecting patients according to the expression level of biomarkers. Further study is needed to explore biomarkers such as PD-L1 expression and TMB in order to identify which patients with this heterogeneous disease are likely to experience greater treatment benefits, so that therapy may be tailored to the individual.

This study has several limitations. First, extrapolations of the survival data were based on the short-term survival data from CASPIAN, and the data would change along with long-term follow-up. As more mature data become available, the model will be more stable. For now, it is an inevitable limitation in our model. Second, immunotherapyrelated AEs are rare, and the cost of treatment in such cases is rather high. Therefore, more cases of immunotherapyrelated AEs would be conducive to more accurate evaluation of $\mathrm{AE}$ cost for patients using durvalumab. In addition, the benefits of durvalumab + chemotherapy would be overestimated in this model. Third, due to the lack of complete quality-of-life data to calculate the utility values, we referred to the mean health utility value of NSCLC in PD, and corrected the utility values by considering the disutility values of AEs, and only grade $3 / 4 \mathrm{AEs}$ were included, which might have led to overestimates or underestimates of the utility values. Finally, the CASPIAN trial did not provide the Kaplan-Meier curve for each subgroup, making it impossible to run the model completely for each subgroup, and the original group balance produced by randomization may not exist in the subgroups; thus, results of the subgroup analyses should be interpreted with caution.

\section{Conclusions}

Our findings showed that durvalumab in combination with platinum-etoposide as first-line treatment is not cost-effective compared with chemotherapy alone in patients with extensive-stage SCLC from the US payer standpoint. 
Submitted August 26, 2020; final revision received October 19, 2020; accepted for publication December 14, 2020.

Published online August 4, 2021.

Author contributions: Study concept and design: Ding, Hu, Huang Performed experiments: Ding, Li, Zhu, Shi, Liao, A. Liu. Data analysis and interpretation: Ding, Hu, Li, Zhu, J. Liu, Tian, Huang. Contributed reagents/ materials/analysis tools: Huang. Manuscript preparation: Ding, Hu, Huang. Final approval of manuscript: All authors.
Disclosures: The authors have disclosed that they have no conflicts of interest with regard to the content of article. This manuscript is original and has not been previously published, nor has it been simultaneously submitted to any other joumal.

Funding: This manuscript was supported by grants from the Hunan Natural Science Foundation of China (No. 2018JJ3852).

Correspondence: Jin Huang, PhD, Department of Oncology, Xiangya Hospital, Central South University, Changsha, Hunan 410008, China. Email: jinhuang@csu.edu.cn

\section{References}

1. Fitzmaurice $\mathrm{C}$, Akinyemiju TF, Al Lami FH, et al. Global, regional, and national cancer incidence, mortality, years of life lost, years lived with disability, and disability-adjusted life-years for 29 cancer groups, 1990 to 2016: a systematic analysis for the global burden of disease study. JAMA Oncol 2018;4:1553-1568.

2. Siegel RL, Miller KD, Jemal A. Cancer statistics, 2019. CA Cancer J Clin 2019;69:7-34.

3. Oronsky B, Reid TR, Oronsky A, et al. What's new in SCLC? A review. Neoplasia 2017;19:842-847.

4. Byers LA, Rudin CM. Small cell lung cancer: where do we go from here? Cancer 2015;121:664-672.

5. Früh M, De Ruysscher D, Popat $S$, et al. Small-cell lung cancer (SCLC): ESMO Clinical Practice Guidelines for diagnosis, treatment and follow-up. Ann Oncol 2013;24(Suppl 6):vi99-105.

6. Rudin $\mathrm{CM}$, Ismaila N, Hann CL, et al. Treatment of small-cell lung cancer American Society of Clinical Oncology Endorsement of the American College of Chest Physicians guideline. J Clin Oncol 2015;33:4106-4111.

7. Rossi A, Di Maio M, Chiodini P, et al. Carboplatin- or cisplatin-based chemotherapy in first-line treatment of small-cell lung cancer: the $\mathrm{COCIS}$ meta-analysis of individual patient data. J Clin Oncol 2012;30:1692-1698.

8. Farago AF, Keane FK. Current standards for clinical management of small cell lung cancer. Transl Lung Cancer Res 2018;7:69-79.

9. Horn L, Mansfield AS, Szczesna A, et al. First-line atezolizumab plus chemotherapy in extensive-stage small-cell lung cancer. N Engl J Med 2018;379:2220-2229.

10. Stewart R, Morrow M, Hammond SA, et al. Identification and characterization of MEDI4736, an antagonistic anti-PD-L1 monoclonal antibody. Cancer Immunol Res 2015;3:1052-1062.

11. Paz-Ares L, Dvorkin $M, C$ Chen $Y$, et al. Durvalumab plus platinumetoposide versus platinum-etoposide in first-line treatment of extensivestage small-cell lung cancer (CASPIAN): a randomised, controlled, openlabel, phase 3 trial. Lancet 2019;394:1929-1939.

12. Loo BW Jr, Akerley W, Bassetti M, et al. NCCN Clinical Practice Guidelines in Oncology: Small Cell Lung Cancer. Version 2.2020. Obtained with permission from NCCN on October 1, 2020. To view the most recent version, visit NCCN.org

13. U.S. Food \& Drug Administration. FDA approves durvalumab for extensive-stage small cell lung cancer. Accessed July 1, 2020. Available at: https://www.fda.gov/drugs/resources-information-approved-drugs/fdaapproves-durvalumab-extensive-stage-small-cell-lung-cancer

14. Goldstein DA, Chen Q, Ayer T, et al. First- and second-line bevacizumab in addition to chemotherapy for metastatic colorectal cancer: a United States-based cost-effectiveness analysis. J Clin Oncol 2015;33:1112-1118.

15. Rini BI, Plimack ER, Stus $V$, et al. Pembrolizumab plus axitinib versus sunitinib for advanced renal-cell carcinoma. N Engl J Med 2019;380:1116-1127.

16. Kohn CG, Zeichner SB, Chen Q, et al. Cost-effectiveness of immune checkpoint inhibition in BRAF wild-type advanced melanoma. J Clin Oncol 2017;35:1194-1202.

17. Nafees B, Lloyd AJ, Dewilde S, et al. Health state utilities in non-small cell lung cancer: an international study. Asia Pac J Clin Oncol 2017;13:e195-203.

18. Yang SC, Kuo CW, Lai WW, et al. Dynamic changes of health utility in lung cancer patients receiving different treatments: a 7-year follow-up. J Thorac Oncol 2019;14:1892-1900.

19. Nafees B, Stafford M, Gavriel S, et al. Health state utilities for non small cell lung cancer. Health Qual Life Outcomes 2008;6:84.
20. Handorf EA, McElligott S, Vachani A, et al. Cost effectiveness of personalized therapy for first-line treatment of stage IV and recurrent incurable adenocarcinoma of the lung. J Oncol Pract 2012;8:267-274.

21. Wan $X$, Zhang $Y$, Tan $C$, et al. First-line nivolumab plus ipilimumab vs sunitinib for metastatic renal cell carcinoma: a cost-effectiveness analysis. JAMA Oncol 2019;5:491-496.

22. Mistry R, May JR, Suri G, et al. Cost-effectiveness of ribociclib plus letrozole versus palbociclib plus letrozole and letrozole monotherapy in the first-line treatment of postmenopausal women with HR+/HER2- advanced or metastatic breast cancer: a U.S. payer perspective. J Manag Care Spec Pharm 2018;24:514-523.

23. Carlson JJ, Canestaro W, Ravelo A, et al. The cost-effectiveness of alectinib in anaplastic lymphoma kinase-positive (ALK+) advanced NSCLC previously treated with crizotinib. J Med Econ 2017;20:671-677.

24. CMS.gov. 2020 ASP Drug Pricing Files. Accessed July 1, 2020. Available at: https://www.cms.gov/medicare/medicare-part-b-drug-average-salesprice/2020-asp-drug-pricing-files

25. von Pawel J, Schiller JH, Shepherd FA, et al. Topotecan versus cyclophosphamide, doxorubicin, and vincristine for the treatment of recurrent small-cell lung cancer. J Clin Oncol 1999;17:658-667.

26. Eckardt JR, von Pawel J, Pujol JL, et al. Phase III study of oral compared with intravenous topotecan as second-line therapy in small-cell lung cancer. J Clin Oncol 2007;25:2086-2092.

27. Ting J, Tien Ho P, Xiang $P$, et al. Cost-effectiveness and value of information of erlotinib, afatinib, and cisplatin-pemetrexed for first-line treatment of advanced EGFR mutation-positive non-small-cell lung cancer in the United States. Value Health 2015;18:774-782.

28. Huang M, Lou Y, Pellissier J, et al. Cost effectiveness of pembrolizumab vs. standard-of-care chemotherapy as first-line treatment for metastatic NSCLC that expresses high levels of PD-L1 in the United States. Pharmacoeconomics 2017;35:831-844.

29. Hoyle M, Green C, Thompson-Coon J, et al. Cost-effectiveness of temsirolimus for first line treatment of advanced renal cell carcinoma. Value Health 2010;13:61-68.

30. Antonia SJ, López-Martin JA, Bendell J, et al. Nivolumab alone and nivolumab plus ipilimumab in recurrent small-cell lung cancer (CheckMate 032): a multicentre, open-label, phase 1/2 trial. Lancet Oncol 2016;17:883-895.

31. Ott PA, Elez E, Hiret $\mathrm{S}$, et al. Pembrolizumab in patients with extensivestage small-cell lung cancer: results from the phase Ib KEYNOTE-028 Study. J Clin Oncol 2017;35:3823-3829.

32. Sequist LV, Chiang A, Gilbert J, et al Clinical activity, safety and predictive biomarkers results from a phase la atezolizumab (atezo) trial in extensivestage small cell lung cancer (ES-SCLC). Ann Oncol 2016;27:493-496.

33. Gadgeel SM, Pennell NA, Fidler MJ, et al. Phase II study of maintenance pembrolizumab in patients with extensive-stage small cell lung cancer (SCLC). J Thorac Oncol 2018;13:1393-1399.

34. Reck M, Luft A, Szczesna A, et al. Phase III randomized trial of ipilimumab plus etoposide and platinum versus placebo plus etoposide and platinum in extensive-stage small-cell lung cancer. J Clin Oncol 2016;34:3740-3748

35. Rudin CM, Awad MM, Navarro A, et al. Pembrolizumab or placebo plus etoposide and platinum as first-line therapy for extensive-stage small-cell lung cancer: randomized, double-blind, phase III KEYNOTE-604 Study. J Clin Oncol 2020;38:2369-2379.

36. Kalemkerian GP, Loo BW Jr, Akerley W, et al. NCCN Clinical Practice Guidelines in Oncology: Small Cell Lung Cancer. Version 1.2019. Obtained with permission from NCCN on October 1, 2020. To view the most recent version, visit NCCN.org 
37. Zhou K, Zhou J, Huang J, et al. Cost-effectiveness analysis of atezolizumab plus chemotherapy in the first-line treatment of extensive-stage small-cell lung cancer. Lung Cancer 2019;130:1-4.

38. Wan X, Luo X, Tan C, et al. First-line atezolizumab in addition to bevacizumab plus chemotherapy for metastatic, nonsquamous non-small cell lung cancer: a United States-based cost-effectiveness analysis. Cancer 2019;125:3526-3534.

39. Ding $\mathrm{D}, \mathrm{Hu} \mathrm{H}$, Liao $\mathrm{M}$, et al. Cost-effectiveness analysis of atezolizumab plus chemotherapy in the first-line treatment of metastatic non-squamous non-small cell lung cancer. Adv Ther 2020;37:2116-2126.

40. Aguiar P Jr, DeMello R, Tadokoro H, et al. O.03: cost effectiveness of immune checkpoint inhibitors in non-small cell lung cancer relative to PD-L1 expression. J Thorac Oncol 2016;11:S169-170.

41. She $L, H u H$, Liao M, et al. Cost-effectiveness analysis of pembrolizumab versus chemotherapy as first-line treatment in locally advanced or metastatic non-small cell lung cancer with PD-L1 tumor proportion score 1\% or greater. Lung Cancer 2019;138:88-94.
42. Insinga RP, Vanness DJ, Feliciano JL, et al. Cost-effectiveness of pembrolizumab in combination with chemotherapy in the 1st line treatment of non-squamous NSCLC in the US. J Med Econ 2018:21:1191-1205

43. Hellmann MD, Ciuleanu TE, Pluzanski A, et al. Nivolumab plus ipilimumab in lung cancer with a high tumor mutational burden. N Engl J Med 2018;378:2093-2104.

44. Yarchoan M, Hopkins A, Jaffee EM. Tumor mutational burden and response rate to PD-1 inhibition. $\mathrm{N}$ Engl J Med 2017;377:2500-2501.

45. Hellmann MD, Callahan MK, Awad MM, et al. Tumor mutationa burden and efficacy of nivolumab monotherapy and in combination with ipilimumab in small-cell lung cancer. Cancer Cell 2019; 35:329.

46. Chung HC, Lopez-Martin JA, Kao CH, et al. Phase 2 study of pembrolizumab in advanced small-cell lung cancer (SCLC): KEYNOTE-158 [abstract]. J Clin Oncol 2018;36(Suppl):Abstract 8506

\section{See JNCCN.org for supplemental online content.}

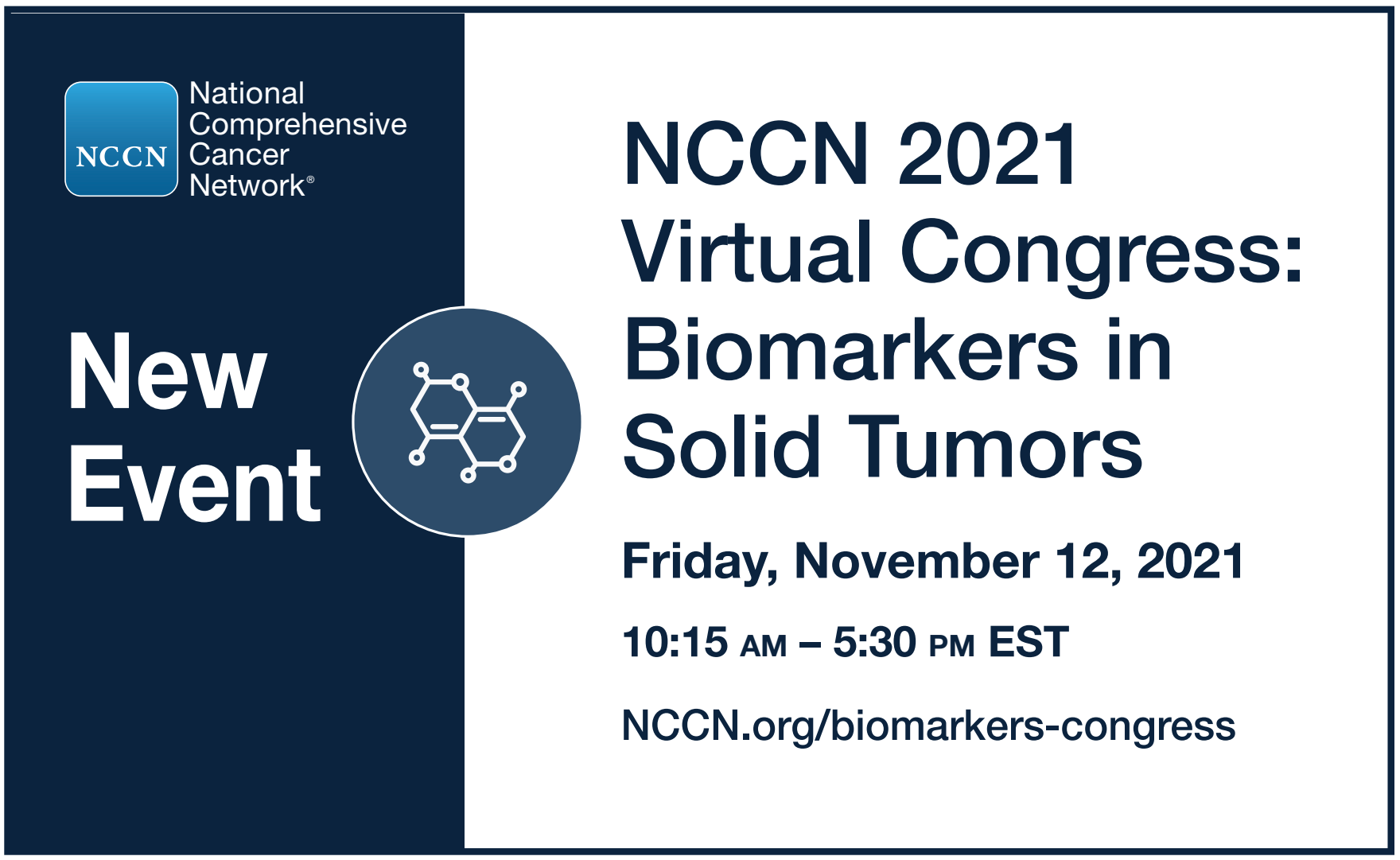


Supplemental online content for:

\section{Cost-Effectiveness Analysis of Durvalumab Plus Chemotherapy in the First-Line Treatment of Extensive-Stage Small Cell Lung Cancer}

Dong Ding, MM; Huabin Hu, MD; Shuosha Li, MM; Youwen Zhu, MM; Yin Shi, MD; Mengting Liao, MD; Jin Liu, MD; Xu Tian, MM; Aiting Liu, MD; and Jin Huang, PhD

J Natl Compr Canc Netw 2021;19(10):1141-1147

eFigure 1: Markov States

eFigure 2: Kaplan-Meier Curve Fitting and Extrapolation

eFigure 3: Results of Monte Carlo Probabilistic Sensitivity Analysis

eTable 1: Summary of Statistical Goodness-of-Fit of Kaplan-Meier Curve in the CASPIAN Trial

eTable 2: Results of Subgroup Analyses 


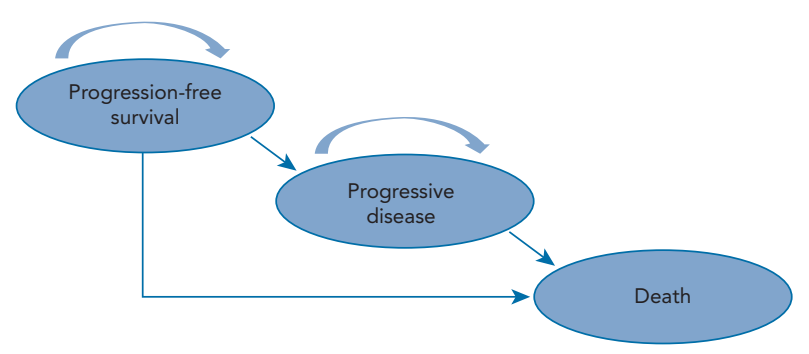

eFigure 1. Markov states, used to illustrate the disease development process of extensive-stage small cell lung cancer.

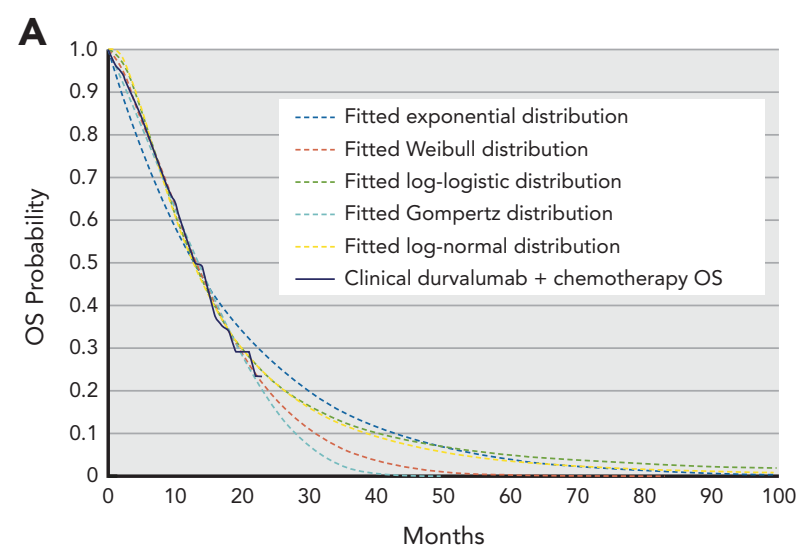

C

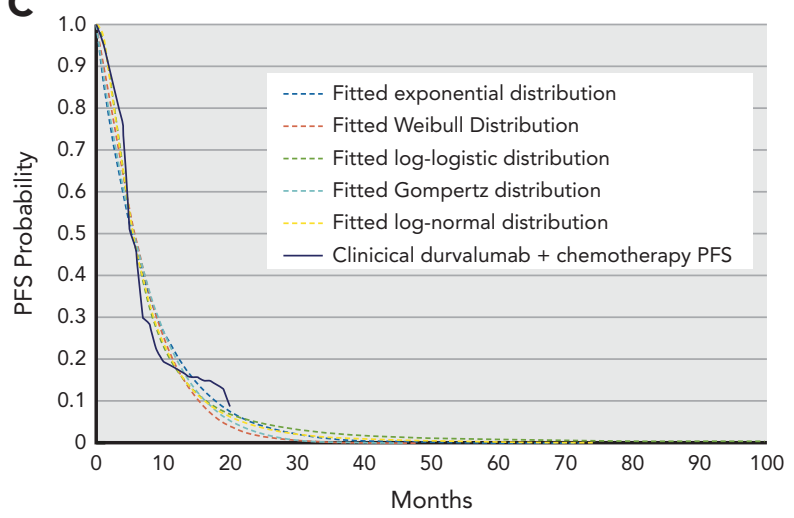

B

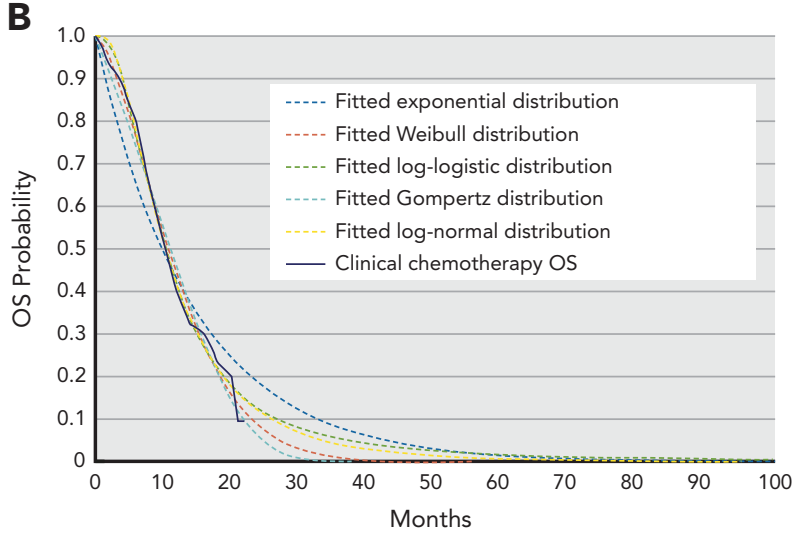

D

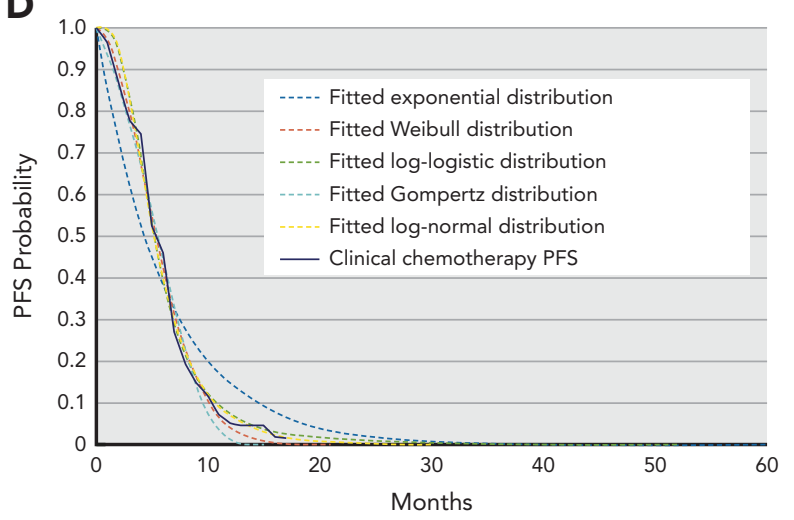

eFigure 2. Kaplan-Meier curve fitting and extrapolation: (A) durvalumab + chemotherapy OS curve; (B) chemotherapy OS curve; (C) durvalumab + chemotherapy PFS curve; (D) chemotherapy PFS curve. Abbreviations: OS, overall survival; PFS, progression-free survival. 


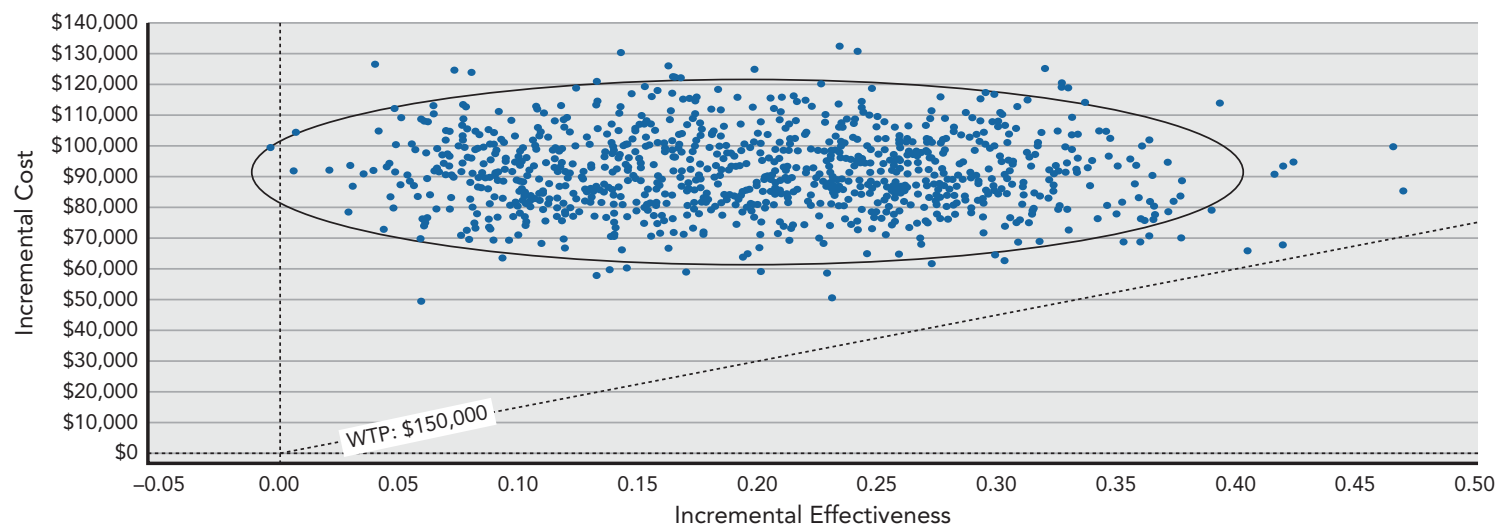

eFigure 3. Results of Monte Carlo probabilistic sensitivity analysis showing incremental cost-effectiveness of durvalumab + chemotherapy versus chemotherapy alone.

Abbreviation: WTP, willingness-to-pay.

\begin{tabular}{|c|c|c|c|c|c|}
\hline & Exponential & Weibull & Log-Logistic & Log-Normal & Gompertz \\
\hline \multicolumn{6}{|c|}{ Durvalumab + chemotherapy OS curve } \\
\hline AIC & 24.3941 & 23.8942 & 23.5959 & 23.5372 & 25.072 \\
\hline $\mathrm{BIC}$ & 26.1667 & 25.4394 & 25.1411 & 25.0823 & 26.9172 \\
\hline \multicolumn{6}{|c|}{ Chemotherapy OS curve } \\
\hline AIC & 27.8527 & 27.6103 & 27.0166 & 26.988 & 27.9779 \\
\hline $\mathrm{BIC}$ & 28.5608 & 28.7264 & 28.4327 & 28.4041 & 29.394 \\
\hline \multicolumn{6}{|c|}{ Durvalumab + chemotherapy PFS curve } \\
\hline $\mathrm{AIC}$ & 47.8501 & 47.4231 & 46.7323 & 46.9699 & 49.5817 \\
\hline $\mathrm{BIC}$ & 49.1581 & 48.8392 & 48.1484 & 48.386 & 50.9978 \\
\hline \multicolumn{6}{|c|}{ Chemotherapy PFS curve } \\
\hline AIC & 53.9253 & 46.5829 & 42.3063 & 44.069 & 91.0773 \\
\hline $\mathrm{BIC}$ & 54.8951 & 47.0678 & 43.2761 & 45.0388 & 92.0471 \\
\hline
\end{tabular}

For the 4 curves listed in the table, the log-logistic and log-normal distribution had the lowest AIC and BIC. Although the AIC and BIC tests are important for determining which models fit the observed data best, they do not tell us how suitable a parametric model is for the time period beyond the final trial follow-up. They described the internal validity of fitted models, but not their external validity. The log-logistic and log-normal distribution produced the longest extended tail in the visual fits of the 4 curves (supplemental eFigure 2), which meant PFS and PFS would be overestimated in long term. Meanwhile, Weibull distributions are flexible and wildly used in cancer survival analyses, and the AIC and BIC of Weibull distribution were slightly higher than that of the log-logistic and log-normal distribution. Weibull distribution was chosen for OS and PFS curves.

Abbreviations: AIC, Akaike information criterion; BIC, Bayesian information criterion; OS, overall survival; PFS, progression-free survival. 


\section{eTable 2. Results of Subgroup Analyses}

\section{Subgroup}

OS HR $(95 \% \mathrm{Cl})$

ICER per QALY $(95 \% \mathrm{CI})$

Cost-Effectiveness Probability at

Planned platinum

\begin{tabular}{llll}
\hline Carboplatin & $0.70(0.55-0.89)$ & $\$ 261,779.97(\$ 210,877.41-\$ 344,058.41)$ & $13.2 \%$ \\
\hline Cisplatin & $0.88(0.55-1.41)$ & $\$ 339,034.60(\$ 210,877.41-\$ 905,637.24)$ & $0.4 \%$
\end{tabular}

Age, y

\begin{tabular}{llll}
\hline$<65$ & $0.74(0.56-0.98)$ & $\$ 277,115.05(\$ 214,002.97-\$ 393,978.41)$ & $7.1 \%$ \\
$\geq 65$ & $0.75(0.54-1.06)$ & $\$ 281,090.44(\$ 207,783.87-\$ 447,218.16)$ & $5.7 \%$
\end{tabular}

Sex

Male $\quad 0.76(0.59-0.97) \quad \$ 285,126.12(\$ 223,582.69-\$ 387,967.19)$

Female $\quad 0.63(0.40-0.98) \quad \$ 236,872.54(\$ 167,275.47-\$ 393,978.41) \quad 20.2 \%$

WHO PS score

\begin{tabular}{llll}
\hline 0 & $0.71(0.48-1.04)$ & $\$ 265,532.25(\$ 189,837.06-\$ 432,970.80)$ & $11.2 \%$ \\
\hline 1 & $0.76(0.59-0.99)$ & $\$ 285,126.12(\$ 223,582.69-\$ 400,120.29)$ & $5.1 \%$
\end{tabular}

Smoking status

\begin{tabular}{llll}
\hline Smoker & $0.72(0.58-0.91)$ & $\$ 269,337.73(\$ 220,354.49-\$ 354,394.51)$ & $7.9 \%$ \\
\hline Nonsmoker & $0.90(0.40-2.11)$ & $\$ 349,177.28(\$ 167,275.47-\$ 433,089.21)$
\end{tabular}

Brain or CNS metastases

Yes $\quad 0.69(0.35-1.31) \quad \$ 258,079.25(\$ 153,780.71-\$ 712,811.73)$

No $\quad 0.74(0.59-0.93) \quad \$ 277,115.05(\$ 223,582.69-\$ 365,137.94) \quad 6.2 \%$

AJCC stage at diagnosis

\begin{tabular}{|c|c|c|c|}
\hline III & $0.92(0.44-1.98)$ & $\$ 359,713.53(\$ 178,385.71-\$ 458,893.47)$ & $0.3 \%$ \\
\hline IV & $0.73(0.58-0.91)$ & $\$ 273,198.08(\$ 220,354.49-\$ 4354,394.51)$ & $8.1 \%$ \\
\hline \multicolumn{4}{|c|}{ Race } \\
\hline Asian & $0.81(0.43-1.49)$ & $\$ 306,278.67(\$ 175,578.43-\$ 1,141,890.97)$ & $3.2 \%$ \\
\hline Non-Asian & $0.73(0.58-0.92)$ & $\$ 273,198.08(\$ 220,354.49-\$ 359,713.53)$ & $6.4 \%$ \\
\hline \multicolumn{4}{|c|}{ Region } \\
\hline Asia & $0.82(0.43-1.54)$ & $\$ 310,719.06(\$ 175,578.43-\$ 135,6795.19)$ & $2.9 \%$ \\
\hline Europe & $0.72(0.56-0.92)$ & $\$ 269,337.73(\$ 214,002.97-\$ 359,713.53)$ & $8.8 \%$ \\
\hline North America and South America & $0.72(0.37-1.44)$ & $\$ 269,337.73(\$ 159,132.32-\$ 982,779.74)$ & $8.8 \%$ \\
\hline
\end{tabular}

Abbreviations: CNS, central nervous system; ICER, incremental cost-effectiveness ratio; LY, life-year; OS HR, overall survival hazard ratio; PS, performance status; QALY, quality-adjusted life-year; WTP, willingness-to-pay. 